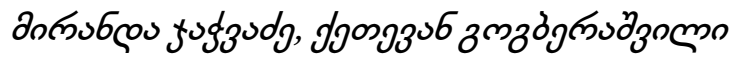

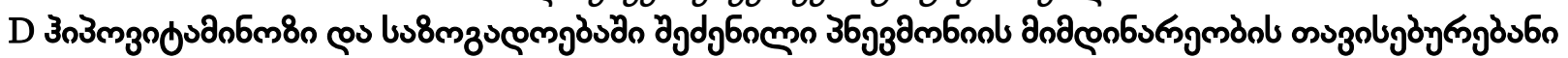

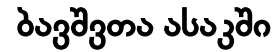

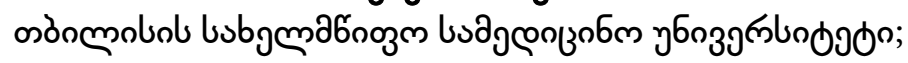 \\ oblin 3. जुs
}

\section{MIRANDA JACHVADZE, KETEVAN GOGBERASHVILI \\ VITAMIN D STATUS AND ITS CORRELATION WITH COMMUNITY ACQUIRED PNEUMONIA SEVERITY IN CHILDREN}

Tbilisi State Medical University; G.Zhvania Pediatric Academic Clinic

\section{SUMMARY}

Objectives. To elucidate the role of vitamin D status on the outcome of pneumonia, we estimated the associations between vitamin D status and pneumonia severity in children. Material and Methods. A hospital based cross-sectional study was conducted in two hospitals. 97 children with community acquired pneumonia (CAP) aged 5-17 years were included in 3 research groups divided according vitamin D status. Vitamin D3 deficiency was defined as a level of serum vitamin $\mathrm{D}<20 \mathrm{ng} / \mathrm{ml}$. The history of the child's illness and the results from the physical examination and laboratory/instrumental data were recorded in a standardized form. Oxygen saturation (SpO2), weight, and height were measured. Indicators of the severity of pneumonia for analysis were presence or absence of danger signs, hypoxemia, prolonged hospitalization, and ICU admission, consolidation on chest X-ray, high inflammatory markers. Statistics. Data analysis was performed using SPSS 18.0 (SPSS Inc., Chicago, IL, USA). The nonparametric tests were used to compare groups (Kruskal-Wallis and MannWhitney). Statistical significance was taken as $\mathrm{p}<0.05$. Results. Children with $25(\mathrm{OH}) \mathrm{D}<20 \mathrm{ng} / \mathrm{ml}$ had an increased risk for treatment failure and a longer duration compared with patients with levels $\geq 30 \mathrm{ng} / \mathrm{ml}$ $(\mathrm{p}<0.001)$. Hypoxemia and respiratory distress syndrome were more common in vitamin D3 deficient patients $(\mathrm{p}<0.05)$. The leukocytosis was in positive correlation with vitamin D3 deficiency, but CRP level was not associated with the vitamin D3 status. Pneumonia cases with 25(OH)D level 20-30ng/ml to compare with vitamin D3 level $>30 \mathrm{ng} / \mathrm{ml}$ cases did not show any significant differences in severity. Conclusion. Vitamin D deficiency (level $<20 \mathrm{ng} / \mathrm{ml}$ ) was associated with severity of CAP in children. There is not yet achieved any consensus about this question and the data from ongoing clinical trials are needed for better elucidation of this matter.

Key words: children, pneumonia severity, vitamin D deficiency

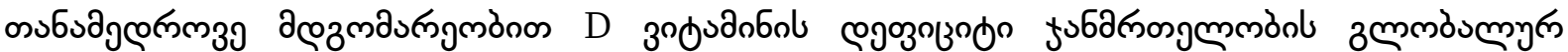

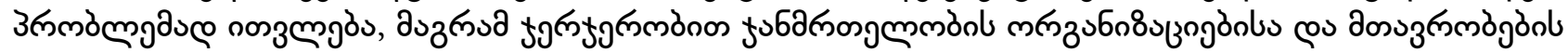

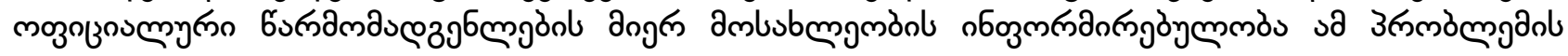

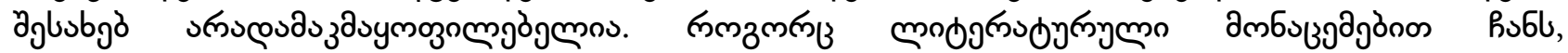

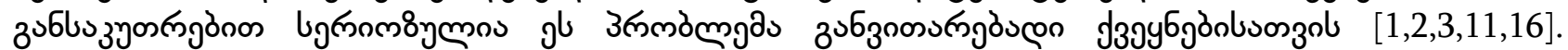

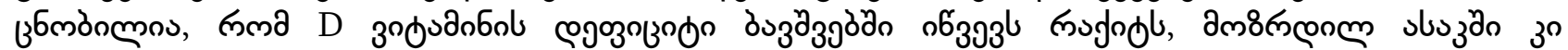

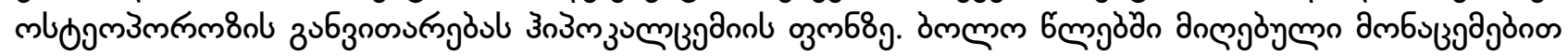

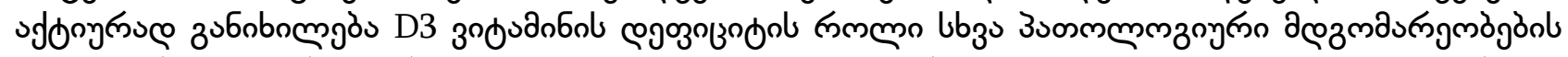

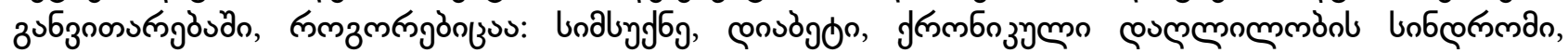

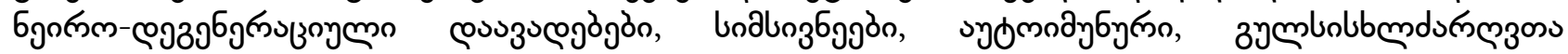

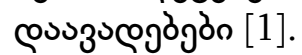

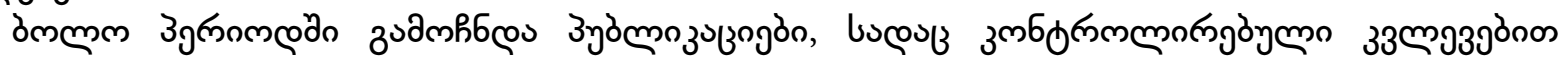

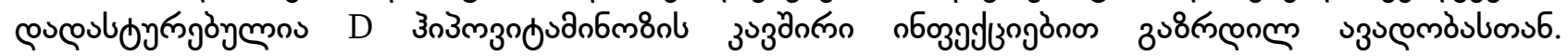

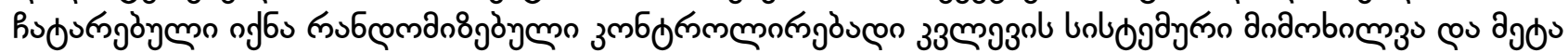

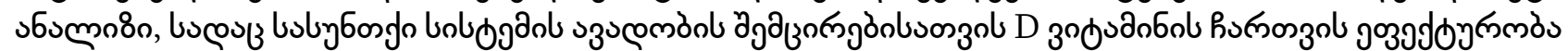

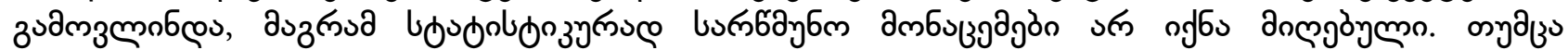

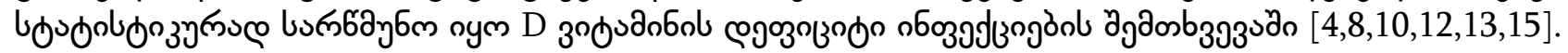

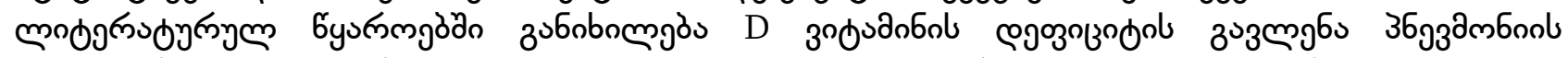

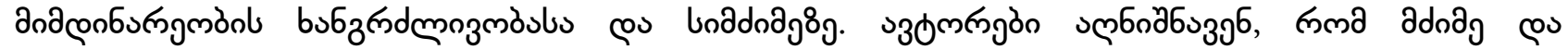

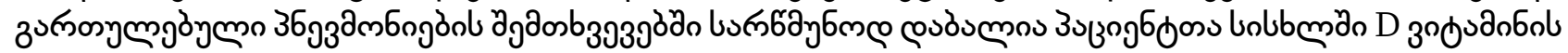


зм6 зэб

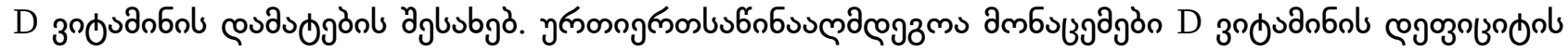

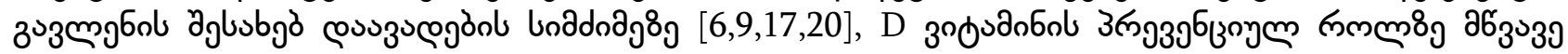

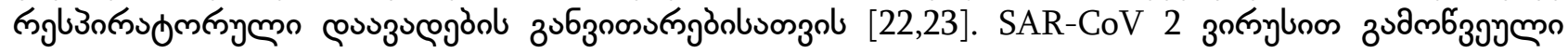

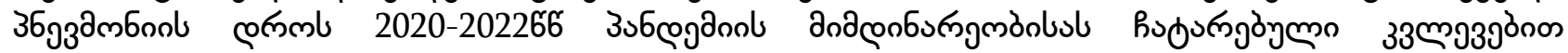

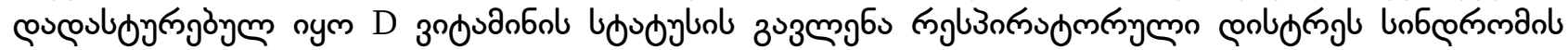

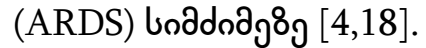

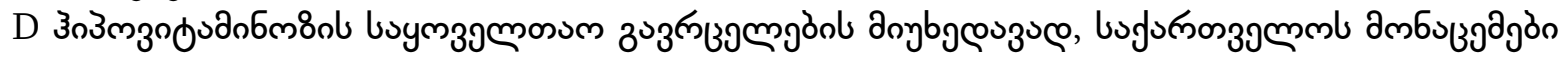

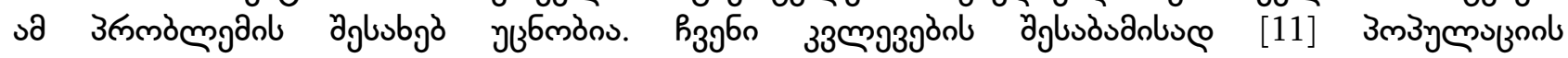

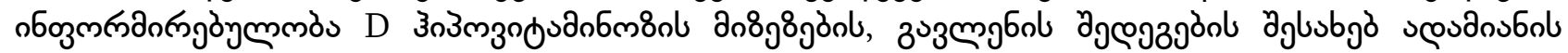

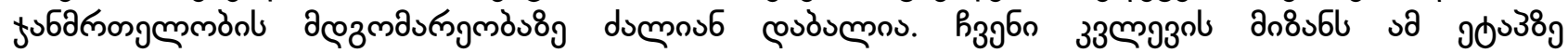

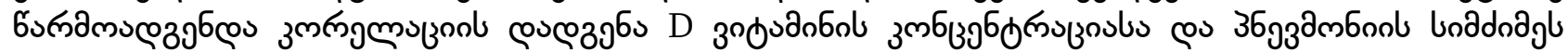

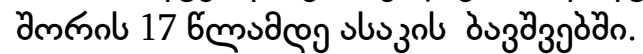

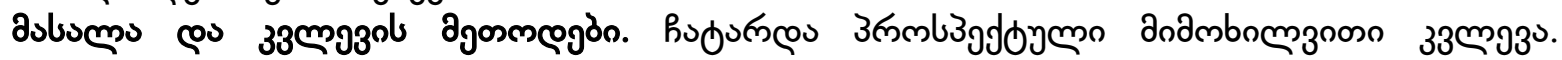

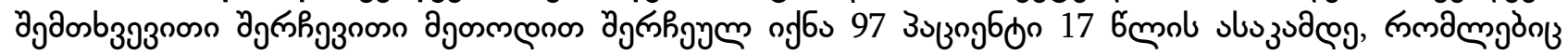

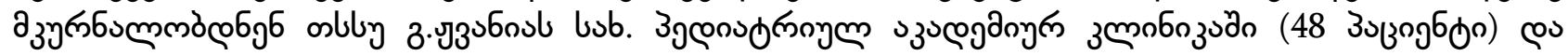

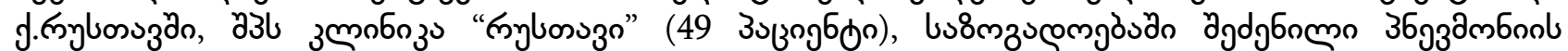

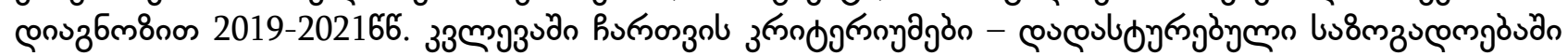

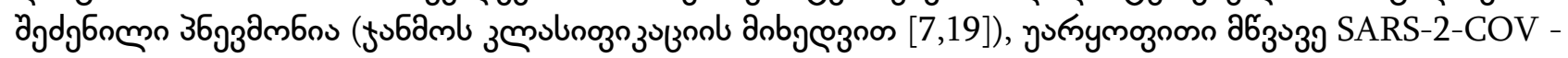

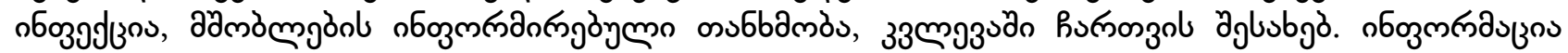

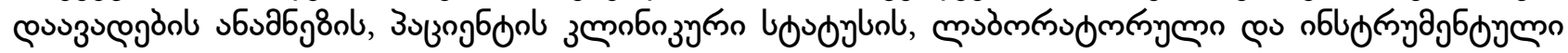

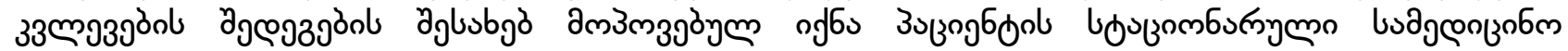

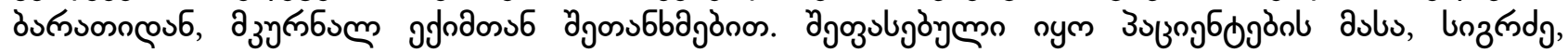

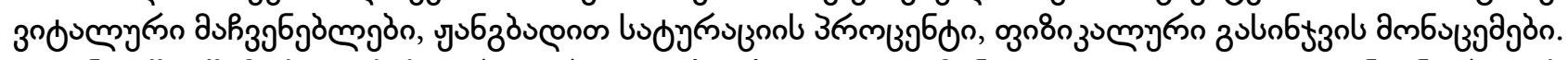

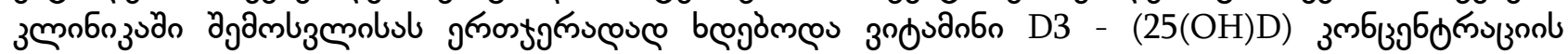
3د6lu8

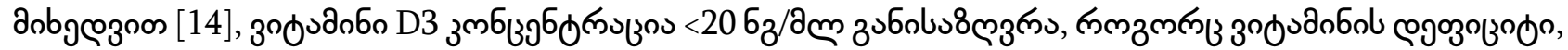

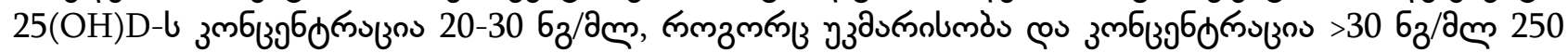

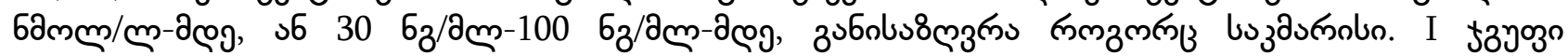

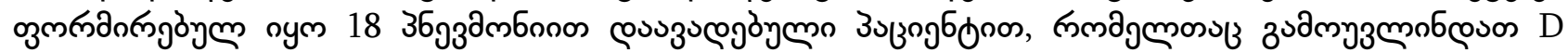

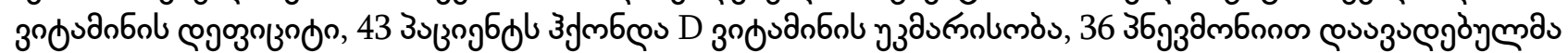

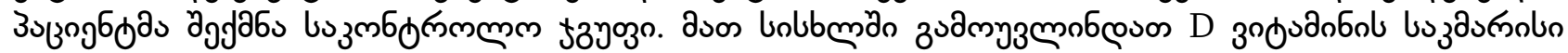

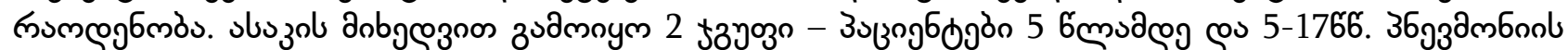

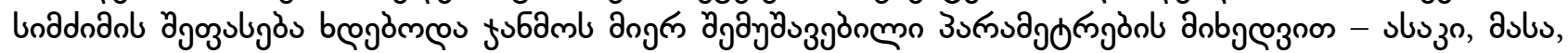

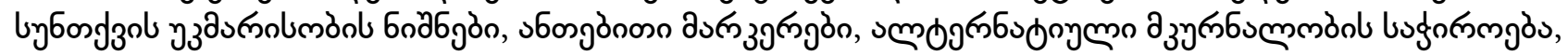

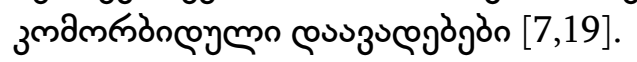

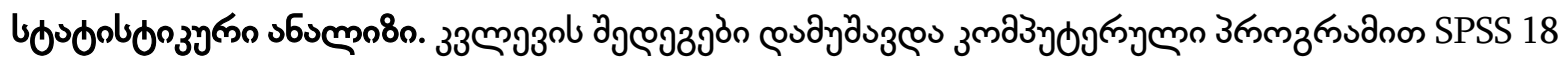

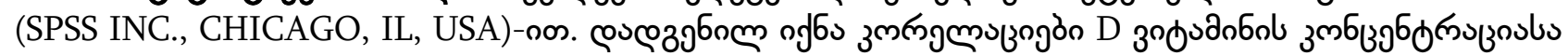

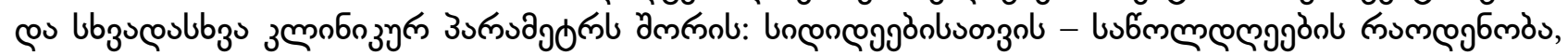

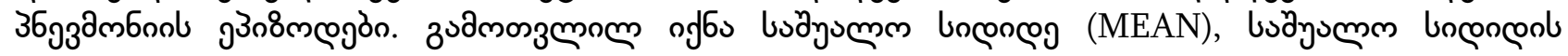

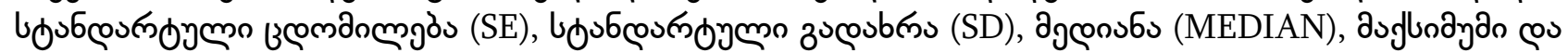

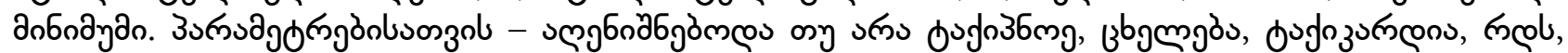

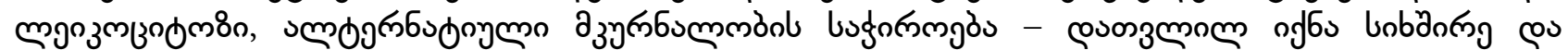

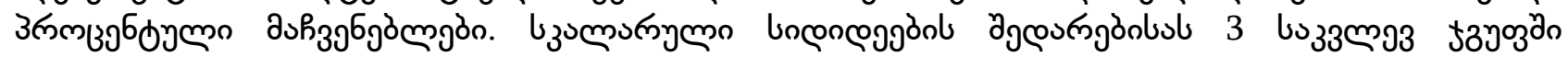

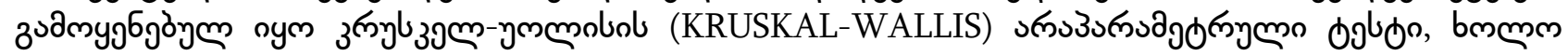

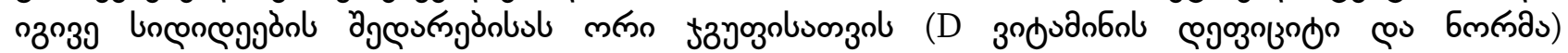

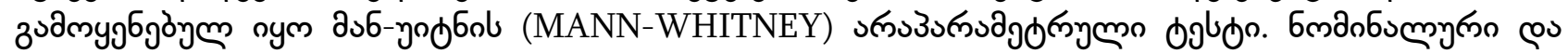

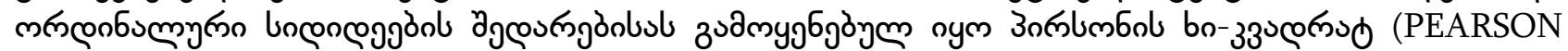

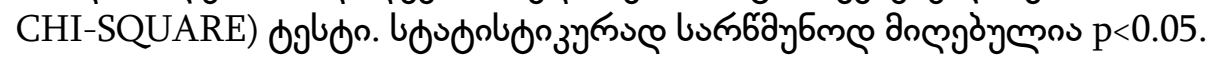

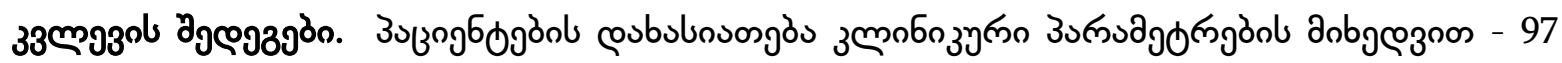

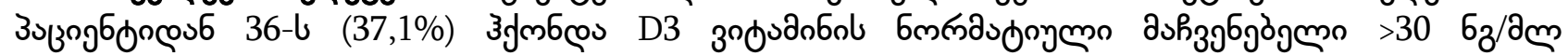




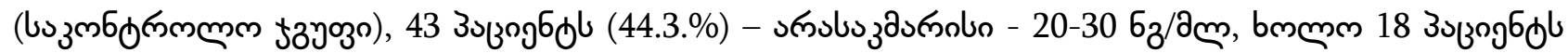

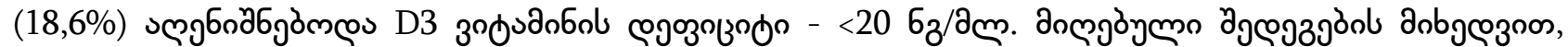
3озоэб

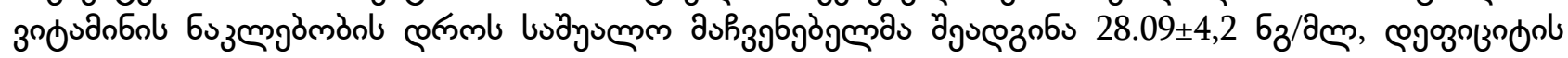

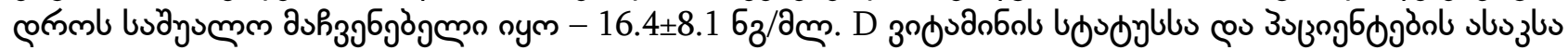

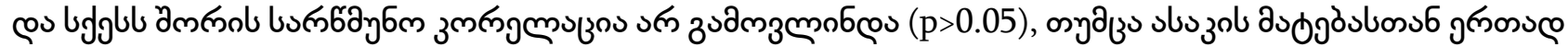

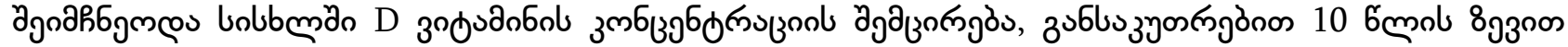

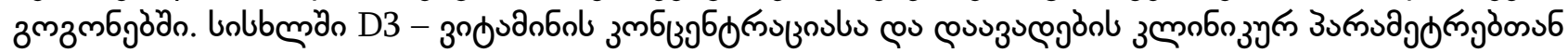

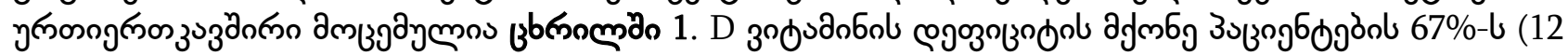

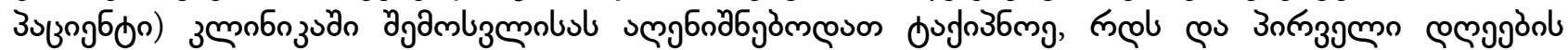

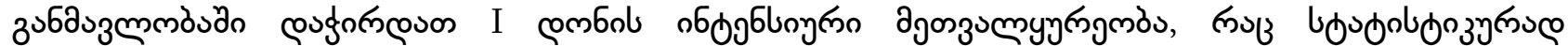

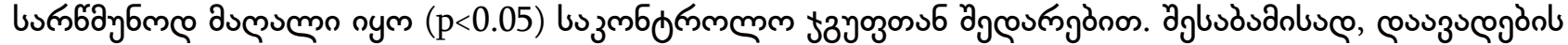

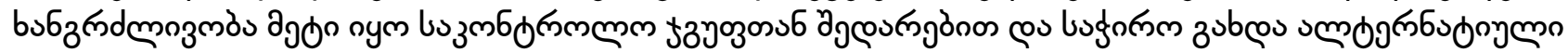

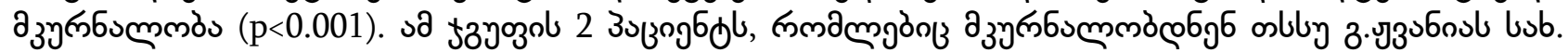

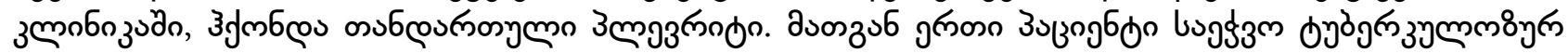

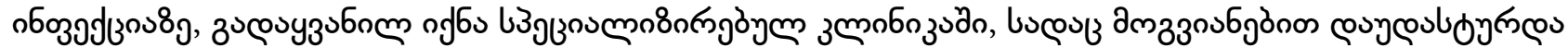

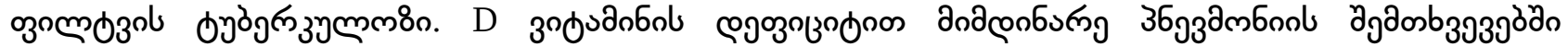

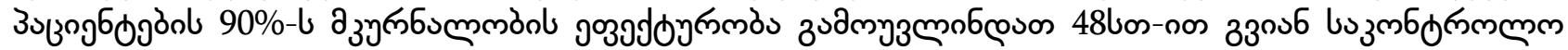

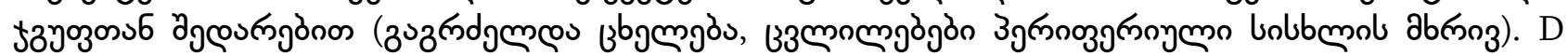

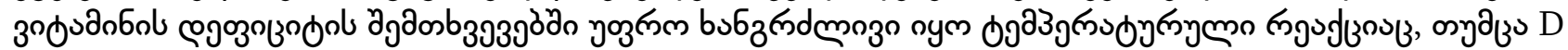

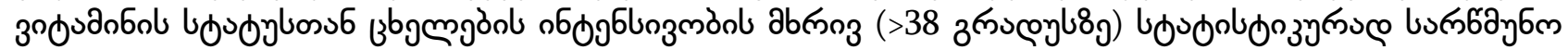

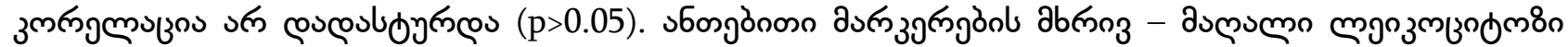

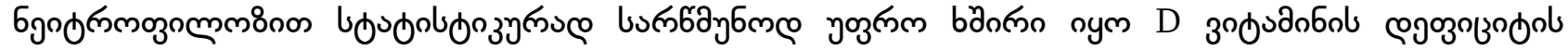

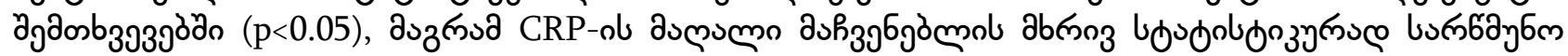

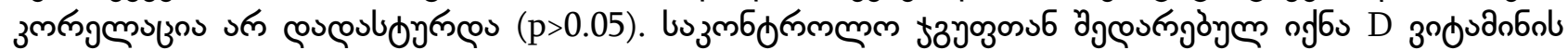

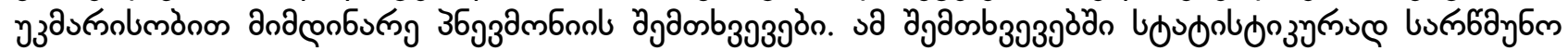

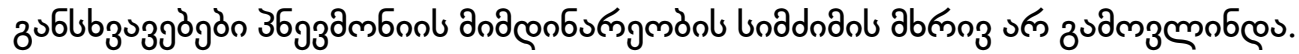

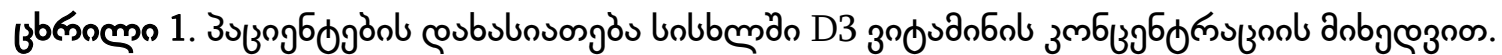

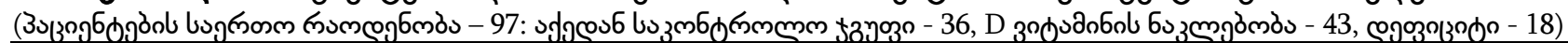

\begin{tabular}{|c|c|c|c|c|c|}
\hline \multirow{2}{*}{ \# } & \multirow{2}{*}{ 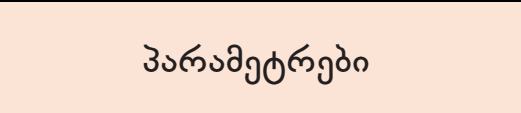 } & 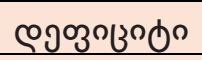 & 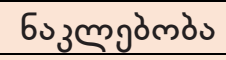 & 6mศృ & \multirow{2}{*}{ lym } \\
\hline & & $<20 \sigma_{3} / \partial m$ & $20-30 \sigma_{3} / \partial m$ & $>30 \sigma_{3} / \partial m$ & \\
\hline \multirow[t]{2}{*}{1.} & ا & $2.8 \%$ & $20.1 \%$ & $22.5 \%$ & \\
\hline & $>5$ 6эмо & $16.2 \%$ & $24.4 \%$ & $14.0 \%$ & $54.6 \%$ \\
\hline \multirow[t]{2}{*}{2.} & 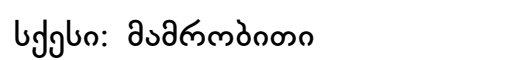 & $17.1 \%$ & $17.1 \%$ & $26.8 \%$ & $61 \%$ \\
\hline & 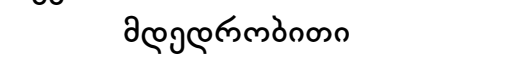 & $9.8 \%$ & $12.2 \%$ & $17.0 \%$ & $39 \%$ \\
\hline \multirow[t]{2}{*}{3.} & 36э3 & & & & \\
\hline & 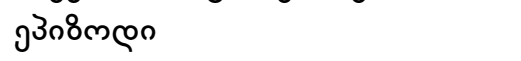 & $22 \%$ & $12.2 \%$ & $4.9 \%$ & $40 \%$ \\
\hline 4. & 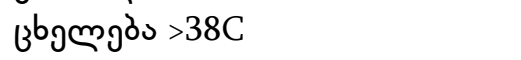 & $17 \%$ & $19.5 \%$ & $41.5 \%$ & \\
\hline 5. & 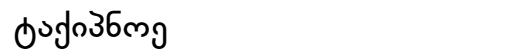 & $24.4 \%$ & $14.6 \%$ & $19.5 \%$ & \\
\hline 6. & 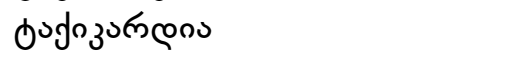 & $24.4 \%$ & $12.2 \%$ & $17.1 \%$ & \\
\hline \multirow[t]{4}{*}{7.} & 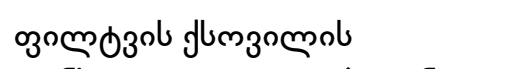 & & & & $100 \%$ \\
\hline & 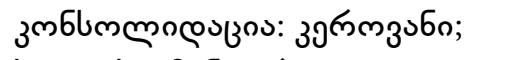 & $6.4 \%$ & $37 \%$ & $37.1 \%$ & $100 \%$ \\
\hline & 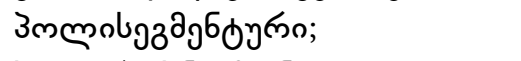 & $4.9 \%$ & $7.3 \%$ & - & \\
\hline & Змэз пмз & $7.3 \%$ & - & - & \\
\hline 8. & 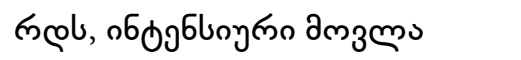 & $9.8 \%$ & $2.4 \%$ & - & \\
\hline \multirow[t]{2}{*}{9.} & 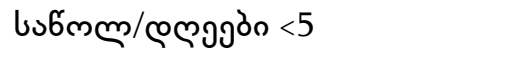 & $0 \%$ & $14.6 \%$ & $31.7 \%$ & \\
\hline & $>7$ & $90 \%$ & $9.8 \%$ & $12.2 \%$ & \\
\hline 10 & 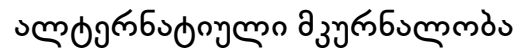 & $14.6 \%$ & - & - & \\
\hline 11 & 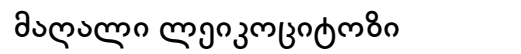 & $19.5 \%$ & $7.3 \%$ & $9.8 \%$ & \\
\hline 12 & 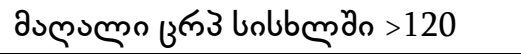 & $9.8 \%$ & $2.4 \%$ & $12.2 \%$ & \\
\hline
\end{tabular}


Әэ५

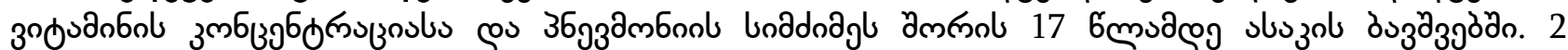

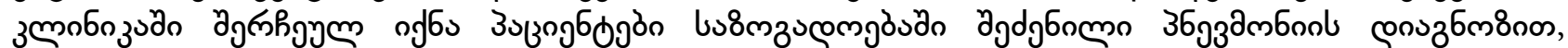

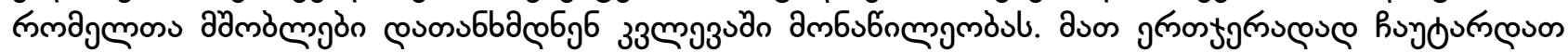
unbbe дे

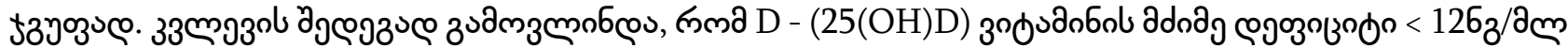
h

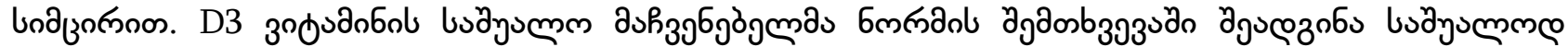

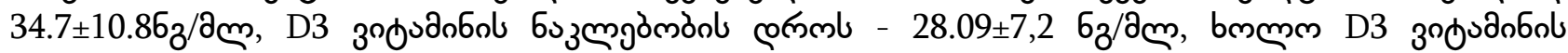

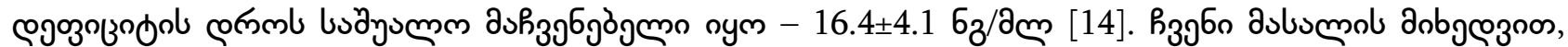

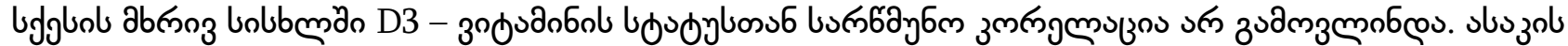

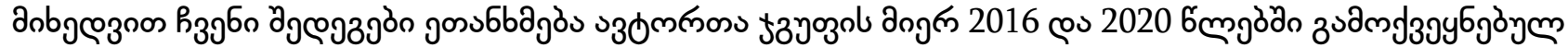

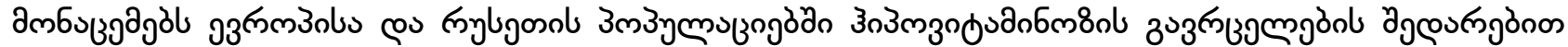

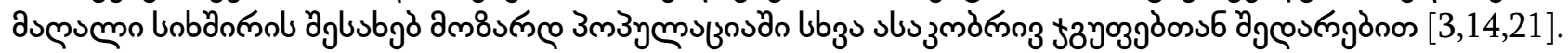

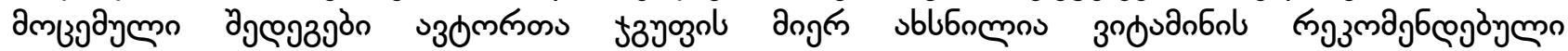

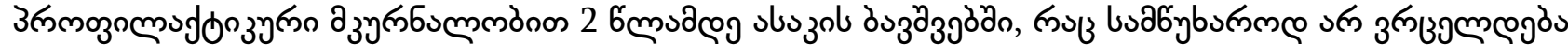

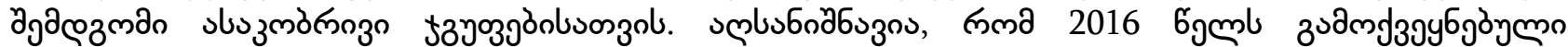

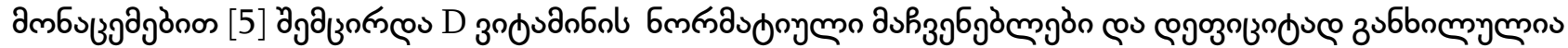
- (25(OH)D) एмб $<306 \partial \mathrm{mm} / \mathrm{m} \nu 6<12 \sigma_{3} / \partial \mathrm{m}$.

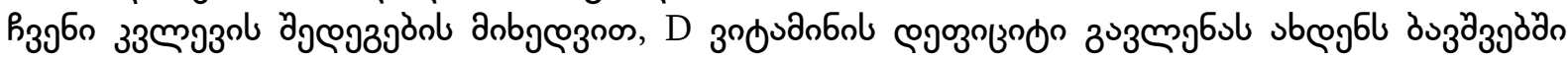

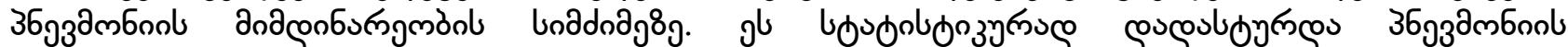

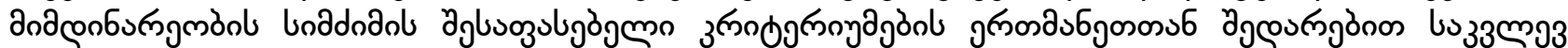

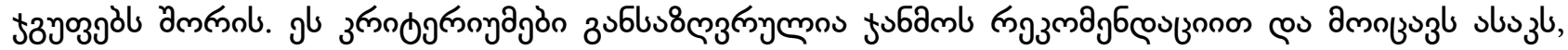

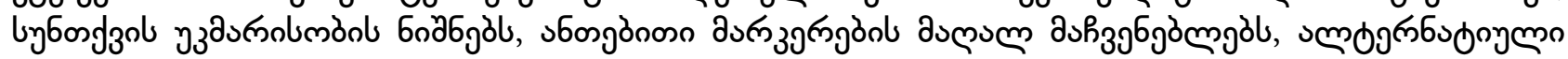

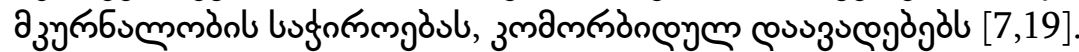

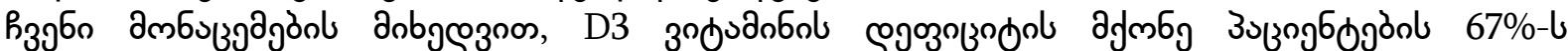
د

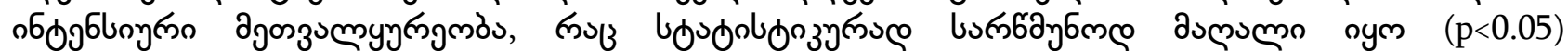

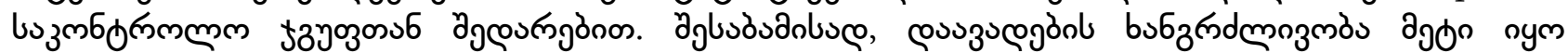

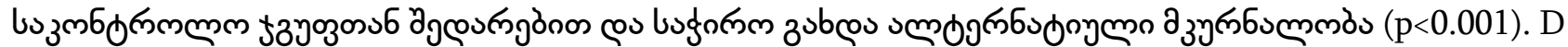

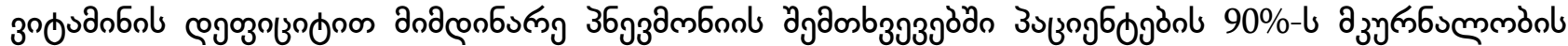

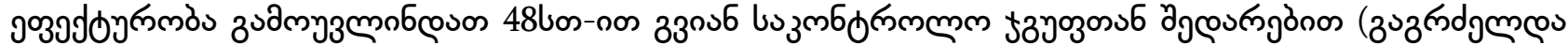
उ उ

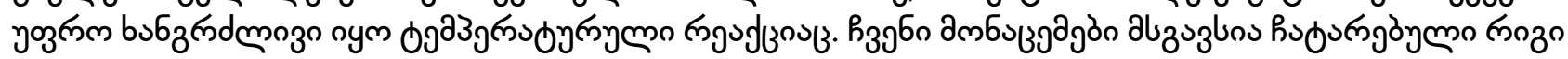

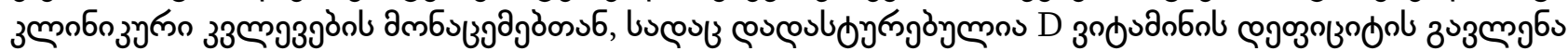
36з3

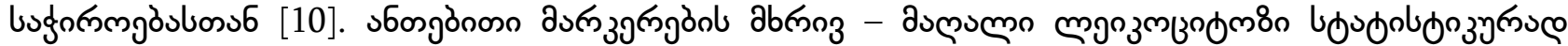

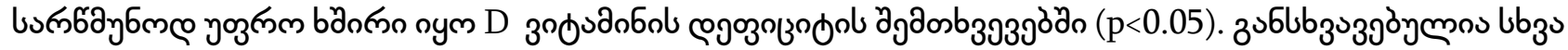

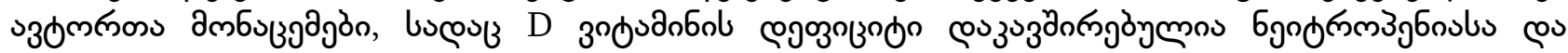

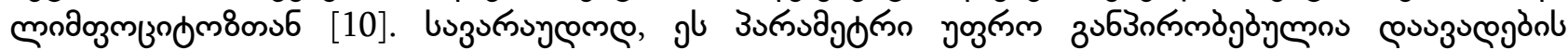

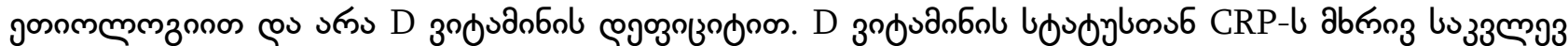

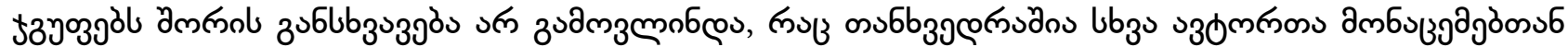
$[9,10]$.

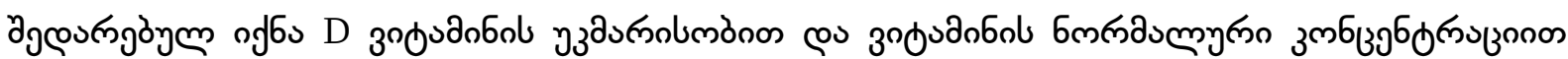

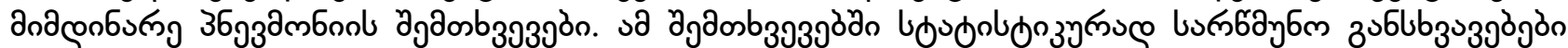

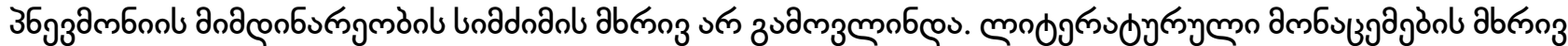
ј9 з з as

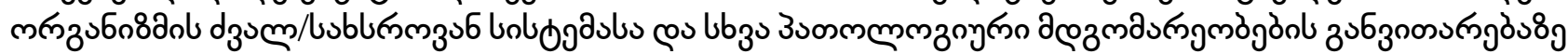

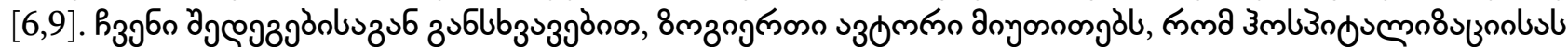

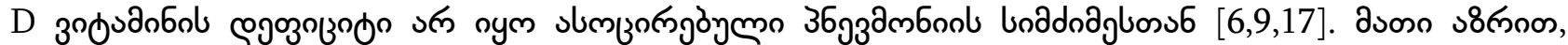

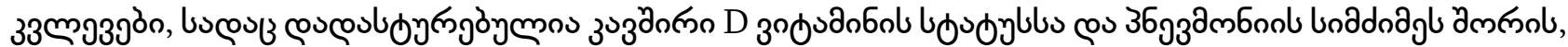




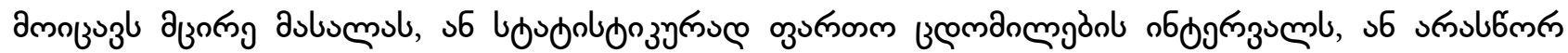

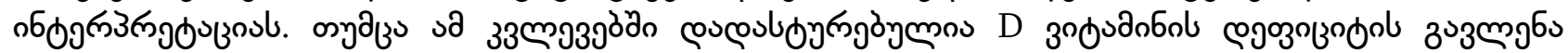

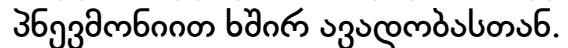

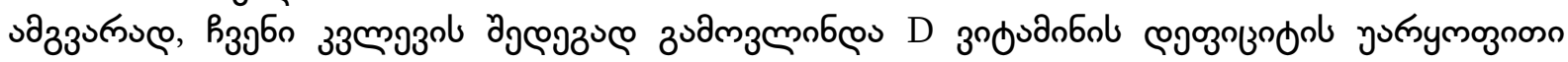

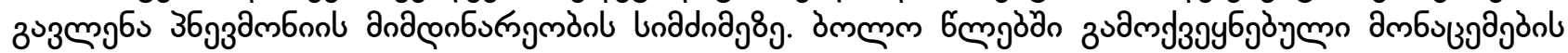

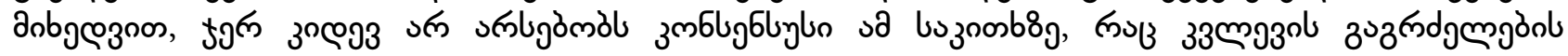

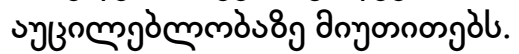

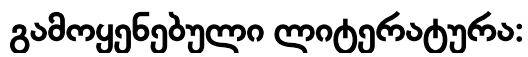

1. Autier P. Mullie P., Macacu A. et al. Effect of vitamin D supplementation on non-skeletal disorders: a systematic review of meta-analyses and randomized trials. Lancet Diabetes Endocrinol 2017; 5: 986-1004.

2. Carr R., Mahmood D., McEvoy A. Vitamin D and Vitamin D Deficiency: How Much Do Parents Know. Arch Dis Child. 2015;100(S3): A84

3. Cashman KD, Dowling KG, Škrabáková Z, Gonzalez-Gross M, Valtueña J, de Henauw S, Moreno L, et al. Vitamin D deficiency in Europe: pandemic? Am J Clin Nutr. (2016) 103: 1033-44. doi: 10.3945/ajcn.115.120873

4. Castillio M.E., Costa L.M.E., Barrios J.M.V., et al. Effect of calcifediol treatment and best available therapy versus best available therapy on intensive care unit admission and mortality among patients hospitalized for COVID-19: A pilot randomized clinical study. www.elsevier.com/locate/jsbmb

5. Craig F. Munns, Nick Shaw, Mairead Kiely, et al. Global Consensus Recommendations on Prevention and Management of Nutritional Rickets. Consensus Statement. J Clin Endocrinol Metab. 2016 Feb; 101(2): 394415.Published online 2016 Jan 8. doi: 10.1210/jc.2015-2175

6. Das RR, Singh M, Naik SS. Vitamin D as an adjunct to antibiotics for the treatment of acute childhood pneumonia. Cochrane Database of Systematic Reviews. 2018(7). pmid:30024634

7. Dean Pr., Florin T.A., Factors Associated with Pneumonia Severity in Children: A Systematic Review. Journal of the Pediatric Infectious Diseases Society, Volume 7, Issue 4, December 2018, Pages 323334, https://doi.org/10.1093/jpids/piy046.

8. Hashemian H, Heidarzadeh A. Role of Vitamin D [25(OH) D] Deficiency in Development of Pneumonia in Children, Arch Pediatr Infect Dis. 2017; 5(3): e57276. doi: 10.5812/pedinfect.57276

9. Haugen J, Chandyo RK, Ulak M, et al. 25-Hydroxy-Vitamin D Concentration Is Not Affected by Severe or NonSevere Pneumonia, or Inflammation, in Young Children. Nutrients 2017:9:52.

10. Haugen J. Basnet S, Hardang IM, et al. Vitamin D status is associated with treatment failure and duration of illness in Nepalese children with severe pneumonia. Pediatr Res. 2017 Dec; 82(6): 986-993. doi: 10.1038/pr.2017.71. Epub 2017 Aug 16.

11.Jachvadze M., Gogberashvili K. Assessment of Knowledge Level Among Georgian Parents About Vitamin D Influence on Child's Health. Questionnaire Survey. GMN, 2020; 10(307):158-161.

12.Jat KR. Vitamin D deficiency and lower respiratory tract infections in children: a systematic review and meta-analysis of observational studies. Trop Doct. 2017 Jan; 47(1): 77-84. Epub 2016 May 13.

13. Kim M., Pfotenhauer DO., Jay H, Shubrook DO. Vitamin D Deficiency, Its Role in Health and Disease and Current Supplementation Recommendations. The Journal of the American Osteopathic Association, May 2017, Vol. 117, 301-305.

14.Kondratyeva E.I., Zakharova I.N., Ilenkova N.A., et al. Vitamin D Status in Russian Children and Adolescents: Contribution of Genetic and Exogenous Factors. Front. Pediatr., 19 November 2020 | https://doi.org/10.3389/fped.2020.583206

15. Martineau AR., Jolliffe DA., Greenberg L., et al. Vitamin D supplementation to prevent acute respiratory tract infections: individual participant data meta - analysis. Health Technol. Assess. 2019 Jan; 23(2): 1-44.

16. Oktaria V, Danchin M, Triasih R, et al. The incidence of acute respiratory infection in Indonesian infants and association with vitamin D deficiency. PLoS One. 2021 Mar 23; 16(3): e0248722. doi: 10.1371/journal.pone.0248722.

17. Oktaria V, Triasih R, Graham SM, et al. Vitamin D deficiency and severity of pneumonia in Indonesian children. PLoS One. 2021 Jul 9; 16(7): e0254488.

18. Quesada-Gomez J.M., Entrenas-Castillo M., Bouillon R. Vitamin D receptor stimulation to reduce acute respiratory distress syndrome (ARDS) in patients with coronavirus SARS-CoV-2 infections: revised Ms SBMB 2020_166, J. Steroid Biochem. Mol. Biol. 202 (2020), https://doi.org/10.1016/j.jsbmb.2020.105719. 
19. Revised WHO classification and treatment of childhood pneumonia at health facilities. Evidence summaries. World Health Organization. Geneva. 2014. Google Scholar.

https://apps.who.int/iris/bitstream/handle/10665/137319/9789241507813 eng.pdf

20. Singh N, Kamble D, Mahantshetti NS. Effect of Vitamin D Supplementation in the Prevention of Recurrent Pneumonia in Under-Five Children. Indian J Pediatr. 2019;86(12):1105-11. pmid:31346969

21.Soliman AT, De Sanctis V, Elalaily R, Bedair S., Kassem I. Vitamin D deficiency in adolescents. Indian J Endocrinol Metab. 2014 Nov; 18(1): S9-S16

22. World Health Organization. Vitamin D supplementation and respiratory infections in children 2019 [updated 29 March 2019; cited 202021 may 2020].

Available from: https://www.who.int/elena/titles/vitamind pneumonia children/en/.

23. Yakoob MY., Salam RA, Khan FR., Bhutta ZA. Vitamin D supplementation for preventing infections in children under five years of age. Cochrane Database Syst Rev 2016; 11: CD008824

\section{МИРАНДА ДЖА ЧВАДЗЕ, КЕТЕВАН ГОГБЕРАШВИЛИ СТАТУС ВИТАМИНА D И ЕГО СВЯЗЬ С ТЯЖЕСТЬЮ ТЕЧЕНИЯ ПНЕВМОНИИ У ДЕТЕЙ}

Тбилисский государственный медицинский университет; ТГМУ Детская академическая клиника им. Г.Жвания

\section{PEЗЮME}

Цель. Выяснить влияние статуса витамина D на исходе пневмонии у детей. Материал и методы. По концентрации витамина D3 в крови 97 детей с пневмонией (BП) в возрасте от 5 до 17 лет были включены в 3 группы. Дефицит витамина D определяли как уровень витамина D в сыворотке <20 нг/мл. Анамнез заболевания ребенка, результаты физикального осмотра и лабораторно-инструментальные данные регистрировались в стандартизированной форме. Измеряли сатурацию кислородом (SpO2), вес и рост детей. Параметрами для определения тяжести пневмонии считались - гипоксемия, длительность госпитализация и лечение в отделение интенсивной терапии, консолидация на рентгенограмме грудной клетки, высокие маркеры воспаления. Статистика. Анализ данных проводили с использованием SPSS 18.0 (SPSS Inc., Чикаго, Иллинойс, США). Для сравнения групп использовались непараметрические критерии (КрускалаУоллиса и Манна-Уитни). Статистическую значимость принимали за $\mathrm{p}<0,05$. Результаты. Дети с уровнем $25(\mathrm{OH}) \mathrm{D}<20$ нг/мл имели повышенный риск неэффективности лечения по сравнению с пациентами с уровнем $\geq 30$ нг/мл (p<0,001). Также, гипоксемия и респираторный дистресс-синдром чаще встречались у пациентов с дефицитом витамина D3 (p<0,05). Лейкоцитоз положительно коррелировал с дефицитом витамина D3, но уровень СРП не был связан со статусом витамина D3. Случаи пневмонии среди детей с недостатком витамина D3 по сравнению со случаями с нормальным содержанием витамина не показали каких-либо существенных различий в степени тяжести. Вывод. Дефицит витамина D3 (уровень <20 нг/мл) ассоциировался с тяжестью пневмонии у детей. По этому вопросу еще не достигнут консенсус, и для лучшего выяснения этого вопроса необходимы дополнительные данные текущих клинических исследовании.

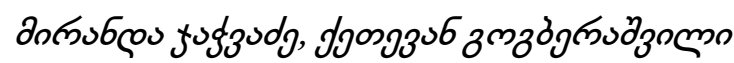

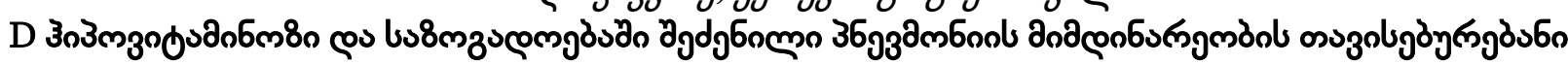

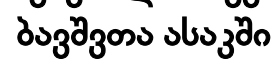

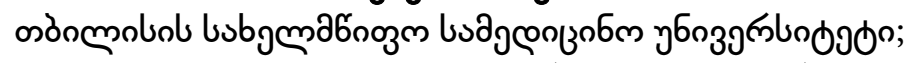

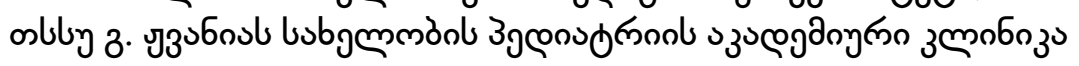

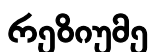

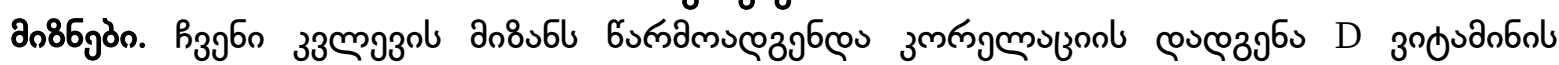

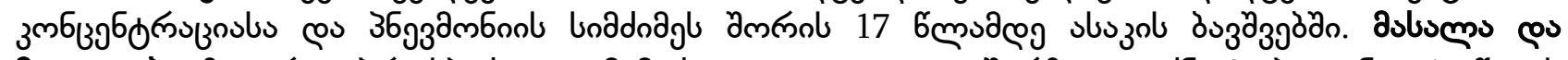

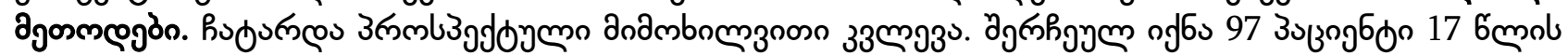

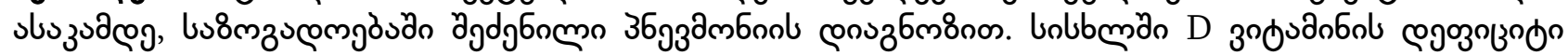




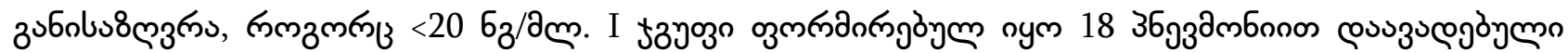

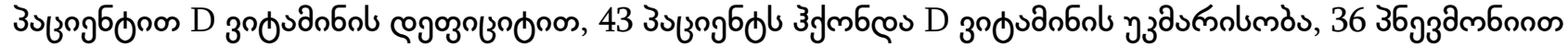

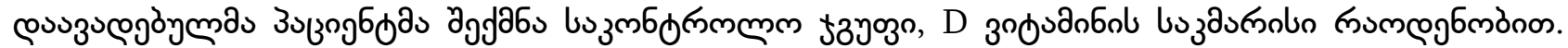
آ

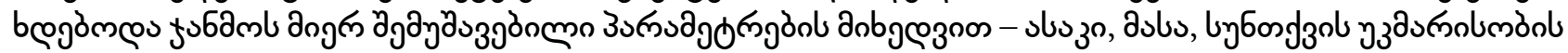

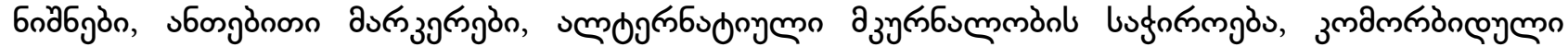

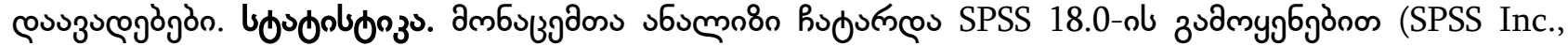

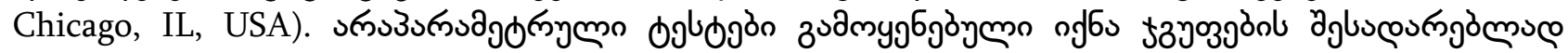

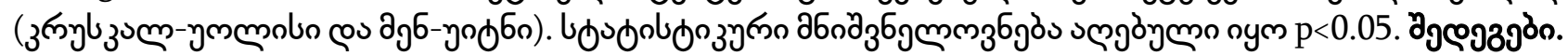

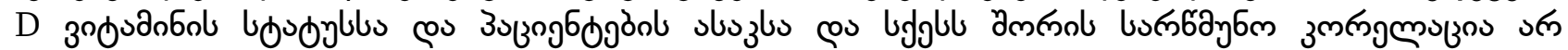

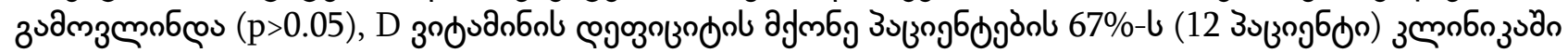

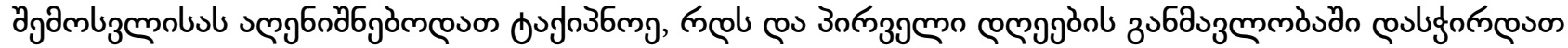

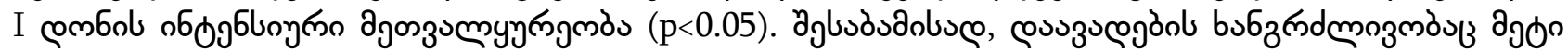

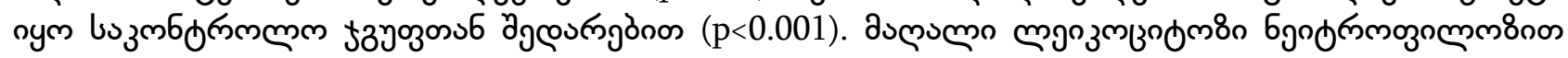

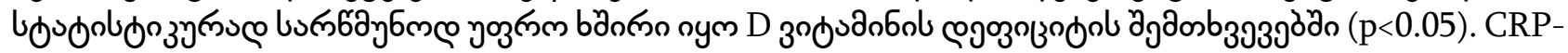
أ

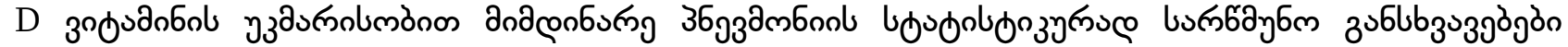

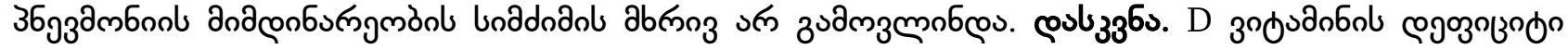

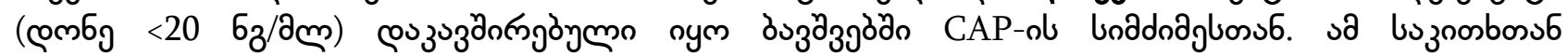

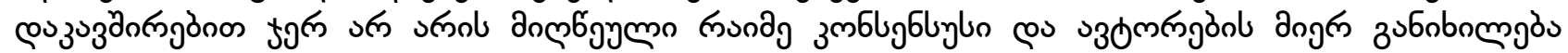

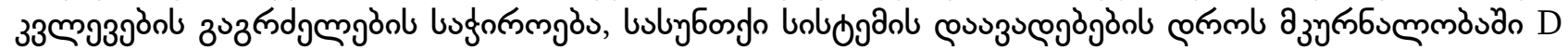

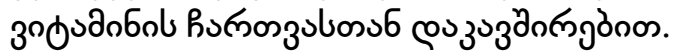

